\title{
Patterns, an efficient way to analyse the p-mode content in rapidly rotating stars
}

\author{
A. García Hernández ${ }^{1, a}$, F. Lignières ${ }^{2}$, L. Balona ${ }^{3}$, D. R. Reese ${ }^{4}$, M. J. P. F. G. Monteiro ${ }^{1}$, J. C. \\ Suárez ${ }^{5}$, and J. Ballot ${ }^{2}$ \\ 1 Instituto de Astrofísica e Ciências do Espaço, Universidade do Porto, CAUP, Rua das Estrelas, \\ PT4150-762 Porto, Portugal \\ 2 Institut de Recherche en Astrophysique et Planétologie (IRAP), CNRS: UMR5277 \\ 3 South African Astronomical Observatory (SAAO), P. O. Box 9 Observatory 7935 Cape Down, South \\ Africa \\ 4 School of Physics and Astronomy, University of Birmingham (UoB), Edgbaston, Birmingham, B15 \\ 2TT, United Kingdom \\ 5 Instituto de Astrofísica de Andalucía (CSIC), CP3004, Granada, Spain
}

\begin{abstract}
High precision photometric observations from space has led to the detection of hundreds of frequencies in the light curves of $\delta$ Scuti pulsators. In this work, we analyzed a sample of Kepler $\delta$ Sct stars to search for frequency patterns in the p-mode regime. To avoid g-modes, we looked at the mode density histogram (MDH). We then used the Fourier transform technique (FT), histograms of frequency differences (HFD) and Echelle diagrams (ED) to find periodicities in the frequency content. We compared the results with those expected for SCF rotating models [4] with the aim of identifying large separations and rotational splittings.
\end{abstract}

\section{Introduction}

The frequency content of $\delta$ Scuti stars is difficult to interpret due to their generally rapid rotation. The large number of frequencies detected from space observations allow the application of statistical techniques to detect frequency patterns [3]. The large separations estimated from such studies are consistent with those predicted by recent self-consistent field (SCF) rotating models [4]. We briefly examine the techniques that can be used to identify frequency patterns in a sample of 15 Kepler $\delta$ Sct stars using KIC 1571717 as an example.

\section{Workflow \& results}

We carefully extracted the frequency content of the light curves following [2] to avoid spurious frequencies. Before searching for patterns, we tried to separate the p- and g-modes regions. We used a MDH diagram (Fig. 1, panel a) to look for the two regions of enhanced mode density separated by a region of low density or a gap. In the simple case of KIC 1571717, both regimes are well separated with a gap around $100 \mu \mathrm{Hz}$ (or $10 \mathrm{~d}^{-1}$ ). We then used the FT technique with the p-modes (as described in [3]) to identify the periodicity corresponding to $\Delta v$ (Fig. 1, b). The prominent peak and its subharmonics suggest that the pattern has a periodicity of $\sim 36 \mu \mathrm{Hz}$ or the double. A HFD of the 30 (or 60 ,

a e-mail: agh@astro.up.pt. AGH acknowledges support from the EC Project SPACEINN (FP7-SPACE2012-312844), from project PTDC/CTE- AST/098754/2008 from FCT (Portugal) and from FCT-MEC (Portugal) through a fellowship (SFRH/BPD/80619/2011).

This is an Open Access article distributed under the terms of the Creative Commons Attribution License 4.0, which permits unrestricted use, distribution, and reproduction in any medium, provided the original work is properly cited. 

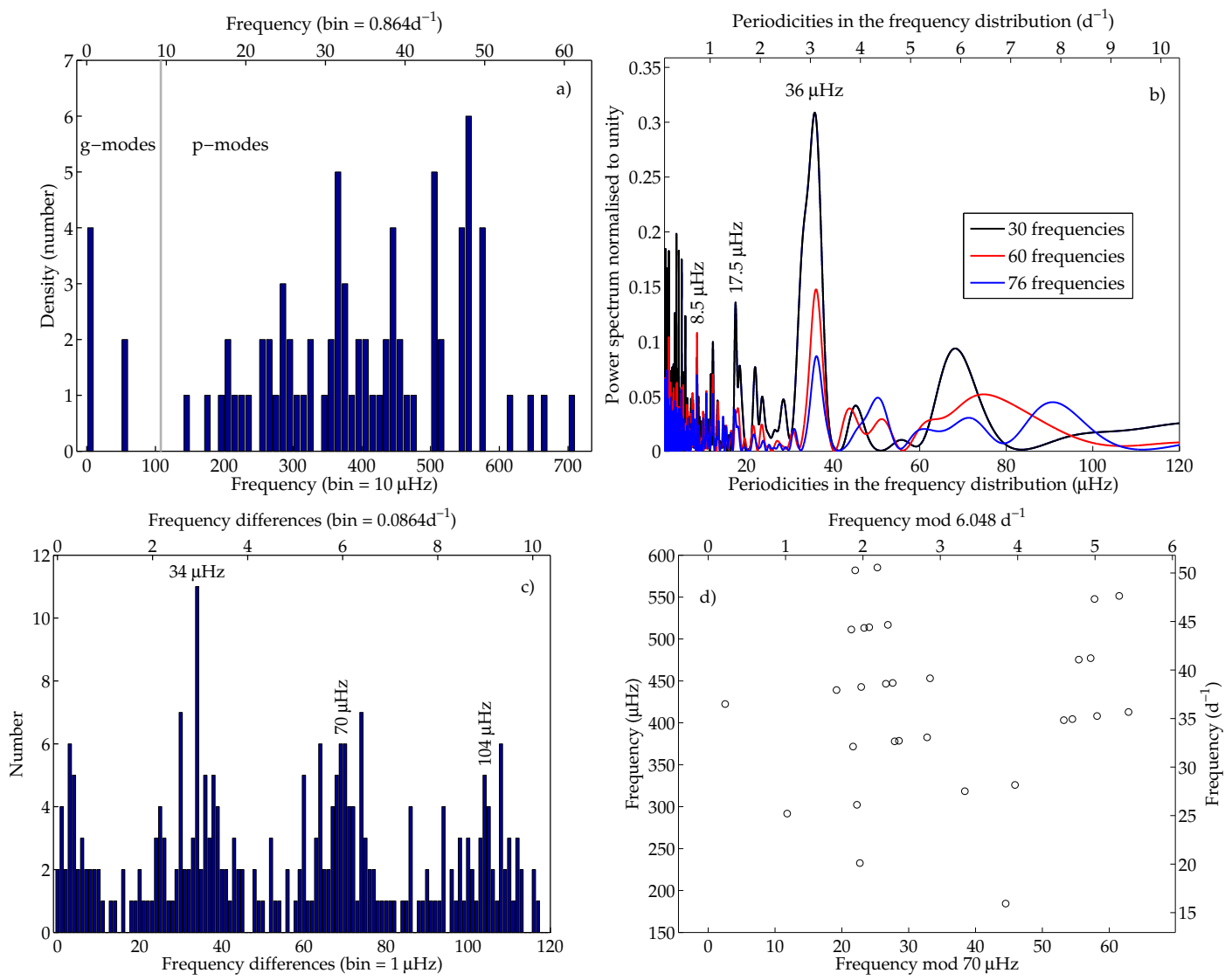

Fig. 1. Example of the workflow followed to find periodicities in the frequency content of $\delta$ Sct stars. The figure shows the case of KIC 1571717. a) MDH to separate the g- and p-mode regimes. b) FT to detect the most probable periodicity. c) Confirmation of the periodicity as a periodic pattern. d) ED showing that $\Delta v$ is indeed the double of the values found in the previous plots.

depending on the case) frequencies with highest amplitudes is used to confirm that the periodicity is, indeed, a large separation as shown by the highly significant peak around the same value and its harmonics (Fig. 1, c). Finally, the ED shows the double column structure expected for a large separation, provided one sets $\Delta v$ to twice the value obtained in the previous tests (Fig. 1, d).

We analysed the sample of 15 Kepler $\delta$ Sct stars following the same procedure. We were able to obtain $\Delta v$ for 11 stars. For some of them, the large separation could be clearly seen in the FT. For the others, we needed to use the HFD to achieve a satisfactory result. Several cases showed a relatively high peak at low frequency differences in the HFD that could be the sign of a rotational splitting. An alternative suggestion supported by statistical studies of Kepler photometry of A stars, is that some peaks at the lower part of the periodogram are compatible with rotation [1]. We would need more precise estimates of the stellar parameters to validate any hypothesis. High resolution spectroscopic observations would be very valuable in this regard.

\section{References}

1. Balona, L. A., MNRAS, 431, (2013) 2240

2. Balona, L. A., MNRAS, 439, (2014) 3453

3. García Hernández, A., Moya, A., Michel, E., et al. A\&A, 559, (2013) A63

4. Reese, D. R., Lignières, F., Ballot, J. et al. A\&A, (2014) submitted 